\title{
La calcolosi urinaria e ill medico di medicina generale
}

\author{
U. Marzocca
}

Medico di Medicina Generale, Firenze

\begin{abstract}
$\boldsymbol{N}$ ella vostra opinione la $N L$ è patologia nella cui gestione è utile l'intervento specialistico?

Ritenete che il nefrologo possa essere il destinatario di parte di questa richiesta?

Nella NL l'approccio può essere solo multidisciplinare; dopo aver eseguito l'ecografia iniziale è necessario indirizzare il paziente al nefrologo, con cui iniziare una coordinata collaborazione.

$\grave{E}$ utile uno studio metabolico dei pazienti con NL? Nella vostra realtà avete accesso a questo tipo di approccio?

Più che utile lo studio metabolico è indispensabile perché permette di comprendere dove intervenire per evitare l'ulteriore crescita del calcolo o prevenire le recidive. Nella nostra realtà di medici di medicina generale possiamo indirizzare i pazienti all' $\mathrm{O}$ spedale "S.M. Annunziata" dell'azienda ASL di Firenze ove vi è la possibilità di realizzare lo studio metabolico.
\end{abstract}

Qual è l'aderenza dei pazienti a un approfondimento diagnostico e a una prevenzione medica della NL nella vostra realtà?

L'aderenza dei pazienti è direttamente proporzionale al tempo che il MMG impiega per spiegare il fine e la realizzazione dello studio metabolico. Inoltre, sarà poi necessario nell'eventualità che lo studio dia risultati positivi, far comprendere al paziente la necessità di assumere, per il resto della vita, quanto necessario a non fare recidivare la nefrolitiasi.

$\grave{E}$ utile, a vostro parere, una profilassi seria delle recidive, basata sulla gestione medica della malattia?

Nella mia esperienza professionale ho constatato che se il paziente segue con diligenza la terapia medica consigliata, anche senza diete particolari, riduce enormemente la probabilità di una recidiva. Questo dimostra, come del resto ci confermano ecografie ed esami radiologici, che i calcoli, se ben trattati, possono anche non crescere o non riformarsi.

Secondo voi dopo venti anni di applicazione della ESWL si sono ottenuti reali vantaggi per il paziente con $N L$ ?

La ESWL è indispensabile per i calcoli già grandi, per risolvere, sia il rischio di infezione, sia il ripetersi di coliche, che alterano la qualità di vita del paziente. Ritengo che qualora il medico di medicina generale di concerto con lo specialista nefrologo intervenisse tramite terapia medica agente sul metabolismo, nei prossimi anni, si potrà sperare di ricorrer sempre meno all'ESWL. 